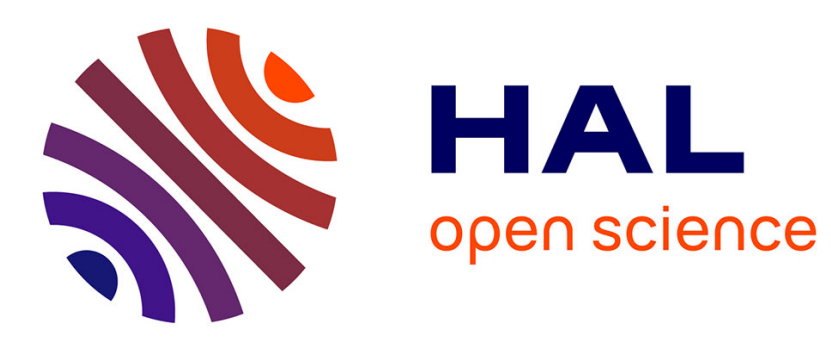

\title{
Comparing position and force control for interactive molecular simulators with haptic feedback
}

Aude Bolopion, Barthelemy Cagneau, Stephane Redon, Stéphane Régnier

\section{To cite this version:}

Aude Bolopion, Barthelemy Cagneau, Stephane Redon, Stéphane Régnier. Comparing position and force control for interactive molecular simulators with haptic feedback. Journal of Molecular Graphics and Modelling, 2010, 29 (2), pp.280-289. 10.1016/j.jmgm.2010.06.003 . hal-00748030

\section{HAL Id: hal-00748030 \\ https://hal.science/hal-00748030}

Submitted on 6 Feb 2013

HAL is a multi-disciplinary open access archive for the deposit and dissemination of scientific research documents, whether they are published or not. The documents may come from teaching and research institutions in France or abroad, or from public or private research centers.
L'archive ouverte pluridisciplinaire HAL, est destinée au dépôt et à la diffusion de documents scientifiques de niveau recherche, publiés ou non, émanant des établissements d'enseignement et de recherche français ou étrangers, des laboratoires publics ou privés. 


\title{
Comparing position and force control for interactive molecular simulators with haptic feedback
}

\author{
Aude Bolopion, ${ }^{* a}$, Barthélemy Cagneau ${ }^{\mathrm{b}}$, Stephane Redon ${ }^{\mathrm{c}}$, Stéphane Régnier ${ }^{\mathrm{a}}$ \\ ${ }^{a}$ Institut des Systèmes Intelligents et de Robotique, Université Pierre et Marie Curie-Paris 6, CNRS UMR 7222, 4 place Jussieu, 75005 Paris, France. \\ ${ }^{b}$ Laboratoire d'Ingénierie des Systèmes de Versailles, EA 4048, Université de Versailles Saint Quentin, 45 avenue des Etats Unis, 78035 Versailles, France \\ ${ }^{c}$ NANO-D - INRIA Rhône-Alpes, 655 avenue de l'Europe, 38334 Saint-Ismier Cedex, France
}

\begin{abstract}
This paper presents a novel tool for the analysis of new molecular structures which enables a wide variety of manipulations. It is composed of a molecular simulator and a haptic device. The simulation software deals with systems of hundreds or thousands of degrees of freedom and computes the reconfiguration of the molecules in a few tenths of a second. For the ease of manipulation and to help the operator understand nanoscale phenomena, a haptic device is connected to the simulator. To handle a wide variety of applications, both position and force control are implemented. To our knowledge, this is the first time the applications of force control are detailed for molecular simulation. These two control modes are compared in terms of adequacy with molecular dynamics, transparency and stability sensitivity with respect to environmental conditions. Based on their specificity the operations they can realize are detailed. Experiments highlight the usability of our tool for the different steps of the analysis of molecular structures. It includes the global reconfiguration of a molecular system, the measurement of molecular properties and the comprehension of nanoscale interactions. Compared to most existing systems, the one developed in this paper offers a wide range of possible experiments. The detailed analysis of the properties of the control modes can be easily used to implement haptic feeback on other molecular simulators.
\end{abstract}

Key words: molecular simulation, haptic coupling, force feedback, transparency, stability

\section{Introduction}

The conception of new medicines, the synthesis of alloys, the prototyping of bio-nanorobots [1] as well as the transmission of information between two nanomachines [2], [3], and the development of bio-microelectromechanical system devices (bioMEMS) [4], [5] are made possible thanks to molecular simulators. Since ease of manipulation is one of the key points of such tools, haptic devices are widely used. They provide a natural manipulation mode of the molecules and force information is easily interpreted by operators. It has been demonstrated that it helps operators to understand nanoscale phenomena. Its use is recommended for educational purposes [6], [7], [8], but it is not limited to academic courses. It can also help chemists and biologists to find specific locations in complex molecular systems (for example docking sites) [9], or to characterize the molecular properties [10].

The benefits of haptic feedback depends on the coupling used. In particular, how to reach the entire virtual environment using a haptic device with a limited workspace is a key issue to get an interactive system. Several techniques are proposed, from the concept of clutching (freezing the displacement of the virtual object while enabling the user to modify the position

${ }^{*}$ Corresponding author: Aude Bolopion, tel: +33144276376, fax: $+33144275145$

Email address: aude.bolopion@isir.upmc.fr (Aude Bolopion) of the haptic handle), to the Bubble technique (position control is used for fine positioning, and rate control is used for large displacements) [11]. As an alternative, the concept of Active Haptic Workspace is considered [12]. As in the Bubble technique position control is used inside the $A H W$, and objects are rotated and translated when the cursor is outside. In all cases, when the user is close to the point of interest, a common position coupling scheme is used to control the object and send the interaction forces to the user. Thus, even if complex techniques are used, the choice of the haptic coupling used remains unchanged when dealing with a precise area of the workspace. The haptic coupling structure depends on the available inputs and outputs. In almost all the works dealing with molecular systems, position control is used (the user sets the position of the molecule, and feels the interaction forces through the haptic interface). However, stability is difficult to ensure due to long computation times, scaling factors used to link the macro and nano worlds, and the high variation of the forces. In most of the works, the system stability is guaranteed at the expense of the fidelity of the force feedback. Either the accuracy of the molecular interactions computed is decreased by using simpler models [13], or the damping added to the coupling deteriorates the transparency [14]. Scaling factors are also a major issue for stability [15]. Position control may also lead to instabilities as the position of the molecule is first set according to the user input, before being corrected during the update step of the simulation using the equation of motion to take into account 
physical constraints, as it is explained in this paper.

In addition to the haptic coupling specificity described above, the system must fulfill several requirements to be usable for the analysis of molecular structures. It should enable the manipulation of the molecules, the measurement of their inherent properties and should provide a deep understanding of the nanoscale interactions. According to potential users (including bio-physicist researchers and researchers working in pharmacological firms), the system should present:

- $R_{1}$ : a fast and accurate manipulation and reconfiguration computation,

- $R_{2}$ : different manipulation choices (both the whole molecule and a single atom), different force feedback (both the internal and external interaction forces),

- $R_{3}$ : transparent and stable haptic coupling schemes despite the computation time.

The system we propose is composed of a molecular simulator, SAMSON, and two haptic coupling schemes. SAMSON enables a fast computation of the interaction forces and of the reconfiguration of the system thanks to a tree representation. The molecules can be manipulated by setting both a position and a force thanks to the methodology used to compute the solution of the equation of motion. Regarding the haptic coupling, a force control scheme is used in addition to position control to compensate for the lack of stability of the position mode while dealing with large displacements. It will also be seen that this control mode is more suited to molecular simulators. Moreover, new applications are possible thanks to this coupling. The control schemes presented are widely used for macroscale teleoperations. Even if force control has already been mentioned for molecular simulation in particular in [16] and [17], to our knowledge, this is the first time it is detailed and analyzed for molecular simulation. It can be implemented in our system thanks to the molecular dynamics equation solved.

We did a preliminary work where some of the potential applications of our system (conception of new molecules, analysis of molecular properties, ...) are described [18]. We now present a comparison of position and force control. A detailed description of the molecular simulator and the control schemes, as well as how they are connected, is made to compare the adequacy of the two control modes to molecular dynamics simulators. The stability sensitivity of the coupling schemes is considered for the specific application of molecular interactions. The sensitivity is defined as the variation of the magnitude of the roots of the control schemes' characteristic equation with respect to given parameters. Relevant experiments of all the stages of the analysis of a molecule are performed and highlight the specificity of each control mode. Based on the experimental results, it seems that the system we propose could be used for the analysis of new molecules, thanks to the two complementary control modes, and to its adequacy with the requirements $\left(R_{1}-R_{3}\right)$.

The rest of the paper is organized as follows. The molecular simulator is presented in Section 2. Based on the available inputs and outputs, the two control modes (position and force control) are detailed and compared in Section 3 in terms of adequacy with the simulator. The transparency and the sensitivity of the coupling schemes' stability is analyzed in Section 4. In Section 5, examples of manipulations that can be handled by our tool are presented. The advantages and drawbacks of each control scheme discussed in previous sections are compared with these experimental data. Section 6 concludes the paper. A table summarizing the notations is provided in Section 7.

\section{Molecular simulator}

As mentioned in Section 1, the molecular simulator must deal with reconfigurable objects of hundreds or thousands of degrees of freedom. Several approaches have been used, such as simplifying the force fields, or considering only rigid molecules. The resulting loss of accuracy limits the possible applications. The simulator we detail hereafter uses a special representation of the molecules, and a specific algorithm based on a quasistatics method to simulate the motion of the molecular system. This leads to a fast computation of both the internal and external forces and a fast reconfiguration of the molecule [19], [20]. Since the simulator has an open architecture, and most functions are implemented as plug-ins, the haptic coupling can be directly integrated into the equations solved by the simulator. This increases the variety of control modes that can be proposed compared to most of the commercially available software.

\subsection{Representation of a molecule}

The method relies on a recursive, divide-and-conquer representation of the molecular system, where any non-rigid group of atoms is considered to be the union of two groups of atoms. Thus, any molecular system is associated to a binary assembly tree, in which the leaf nodes represent the user-defined rigid bodies (atoms, or group of atoms). The internal nodes represent the sub-assemblies, and the root node represents the complete molecular system (Figure 1).

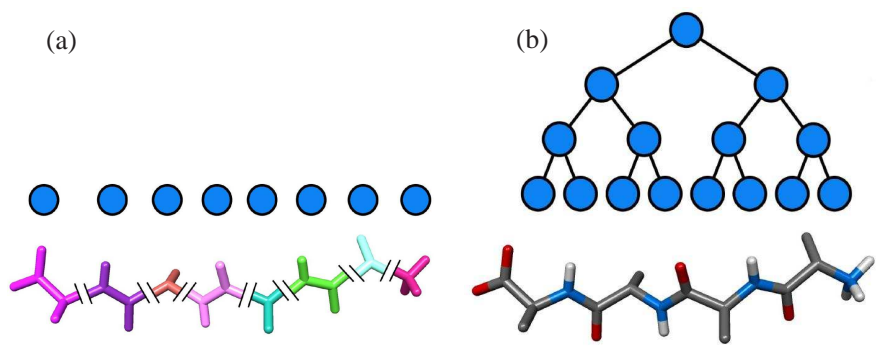

Figure 1: Representation of the tetra-alanine molecule. (a): The leaf nodes represent the user defined rigid bodies, (b): The binary assembly tree where the internal nodes represent the sub-assemblies, and the root node represents the complete molecular system.

Using the spatial notation, the equation of motion of each rigid body is [21]:

$$
I \cdot a=F_{\text {ext }}+F_{\text {int }}-v \times I \cdot v
$$


where $a$ (resp. $v$ ) is the spatial acceleration (resp. velocity) of the molecule. $I$ is its inertia. $F_{\text {ext }}$ is the sum of the forces applied by other molecules and $F_{\text {int }}$ is the sum of the internal forces applied to the considered rigid body. These forces depend on the force field being used in the simulation to model interatomic interactions. In our current implementation, the force field is derived from a well known molecular mechanics force field, CHARMM [19], which models interactions through van der Waals, electrostatic and dihedral contributions.

\subsection{Computation of the solution of the equation of motion}

To solve the equation of motion for each rigid body, the simulator follows traditional approaches: at each time step, the interatomic forces are first computed, then the acceleration of the system is determined and used to update its state and the internal configuration of the molecule. This operation is made easier thanks to the tree representation, which enables to recursively compute the forces and accelerations from the root node to the leaves after having recursively computed the coefficients of the equation in a bottom-up pass. Thanks to the chosen representation of the molecule, and the way of solving the equation of motion, systems of hundreds of degrees of freedom can be simulated in tenths of a second.

\subsubsection{Manipulation modes}

To enable the user to manipulate the molecule (or one of the rigid bodies), its action must be taken into account while solving the equation of motion. Thanks to the open architecture of the software, two solutions are possible. They are detailed hereafter:

Position mode. One way to control the simulation through the haptic device is to control the position of the manipulated rigid body or molecule. In the algorithm, the instantaneous position is set by the user, instead of being determined by the computed acceleration. The internal degrees of freedom are simulated using Equation (1). This manipulation mode is intuitive for the user, since we are used to set the position of macroscopic objects. However, between two simulation steps, the position set by the user may be physically unacceptable (e.g. atom clashes may happen).

Force mode. Another way to control the simulation is to apply a force on the manipulated objects. This force $F_{i}$ is added to the interatomic forces that are applied to the object, and the total force is used to determine the accelerations in the molecular system and update the positions of the rigid bodies. The equation of motion is then:

$$
I \cdot a=F_{i}+F_{e x t}+F_{\text {int }}-v \times I \cdot v
$$

Based on these manipulation modes, two corresponding control schemes may be used for position and force control.

\subsubsection{Force sent to the user}

The simulator determines the force $F_{m}$ applied to the manipulated molecule. It is composed of van der Waals and electrostatic contributions from other molecules $F_{\text {ext }}$, as well as those resulting from other rigid bodies in the selected molecule $F_{\text {int }}$ :

$$
F_{m}=F_{\text {ext }}+F_{\text {int }}
$$

The user will thus feel both internal and external forces. Note that, in this sum, the contributions from rigid bodies within the selected molecule cancel out if the user manipulates a whole molecule $\left(F_{\text {int }}=\sum F_{\text {int }}^{j}=0\right)$. When only one rigid body from a molecule is selected, the molecular force $F_{m}^{j}$ applied to the $j^{\text {th }}$ rigid body by the environment is computed as before, but the kinematic constraint forces, i.e. the forces which maintain the kinematic constraints between the selected rigid body and its topological neighbors [19] do not cancel out. In particular, if the rigid body is part of a molecule on which no external force is applied, the user will only feel the kinematic constraints. Since no modification on our system are necessary the user can easily switch from one mode to the other.

Note that, although we have used SAMSON simulator in our implementation, the control schemes we will present may be connected with any simulator which provides access to the required variables (positions, forces, etc.).

\section{Coupling between the simulation software and the haptic interface}

Before considering the two control schemes some notations must be introduced for both the simulator and the haptic coupling. These notations are summarized in Section 7.

\subsection{Notations}

\subsubsection{Simulator}

The $n$ rigid bodies (atoms or groups of atoms) which are inside the manipulated molecule will be noted $\mathcal{R}_{m}^{j}, j=1, \ldots, n$ and the $q$ ones which are part of the environment will be noted $\mathcal{R}_{e}^{l}$, $l=1, \ldots, q$ (see Figure 2).

The force applied to each rigid body $\mathcal{R}_{m}^{j}$ by all the other rigid bodies of the simulation (including internal forces $F_{i n t}^{j}$ and external forces $F_{\text {ext }}^{j}$ ) is: $F_{m}^{j}=F_{\text {int }}^{j}+F_{\text {ext }}^{j}$. The total force applied to the manipulated molecule $F_{m}$ is: $F_{m}=\sum_{j} F_{m}^{j}$. The position of $\mathcal{R}_{m}^{j}$ after the reconfiguration step is $x_{m}^{j}$, and $x_{m}$ is the position of the center of mass of the manipulated molecule. Similarly, $F_{e}^{l}=F_{i n t}^{l}+F_{e x t}^{l}$ is the force applied to each rigid body $\mathcal{R}_{e}^{l}$ by all the other rigid bodies of the simulation, $x_{e}^{l}$ is its position after the reconfiguration step. $m_{p}$ is the mass of the $p^{\text {th }}$ rigid body in the simulation. $m$ is the total mass of the manipulated molecule. The molecular dynamics do not allow to use the same time basis as the one we use [16]. A single simulation step takes around 100 milliseconds (moving the molecule, reconfiguring the system, sending the interaction forces to the user), and this corresponds to a period of around $T_{m}=2$ femtoseconds for the evolution of the molecular system. The molecular integration time $T_{m}$ is thus introduced. 


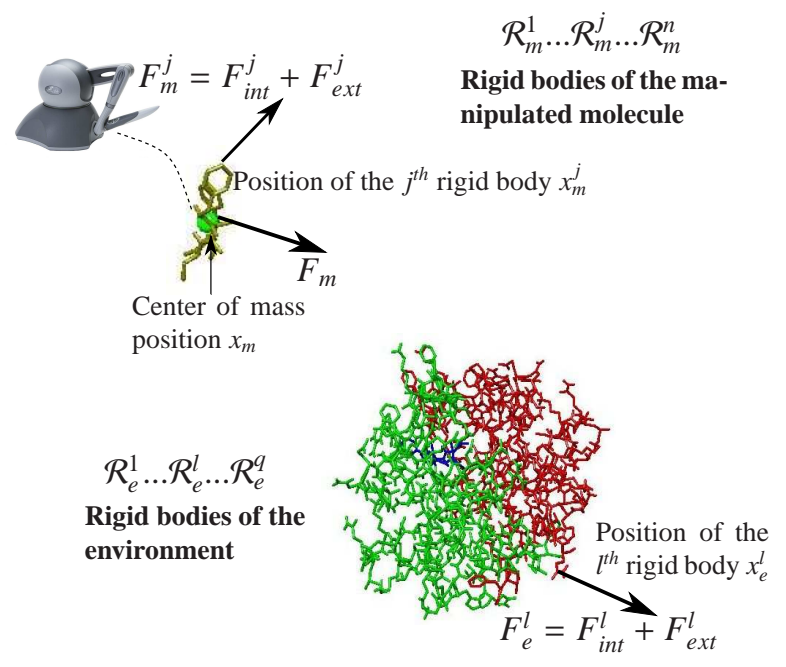

Figure 2: Notations related to the molecular simulator

\subsubsection{Haptic coupling}

When connecting a haptic (or master) device to a molecular simulator, the delays due to long computations of complex interactions must be taken into account while analyzing the stability of the control scheme. The transfer functions are thus represented using the discrete time variable $z$. Since the variables coming from the simulator are delayed by one sampling period $T_{s}$ (defined as the largest simulation period for a given experiment), an explicit one step delay is introduced between the simulation and the coupling. Note that we consider here the worst case by taking the largest delay. This is modeled by the delay operator $z^{-1}$ (see Figure 3 and Figure 4). The discrete time transfer function $H(z)$ of the haptic interface (which input, resp. output, is the force applied to the haptic handle, resp. its position) is computed from the continuous time domain using the Z-transform function [22], and taking into account the effects of the sampler and zero order hold:

$$
H(z)=\frac{1}{B_{h}} \frac{T_{s}(z-\delta)-(1-\delta)(z-1) \frac{M_{h}}{B_{h}}}{(z-1)(z-\delta)}
$$

where $\delta=e^{-\frac{B_{h} T_{s}}{M_{h}}} . M_{h}$ and $B_{h}$ are respectively the master's inertia and viscosity, as given in [23].

Due to the scale difference between the macro and the nanoworlds, two scaling factors must be introduced to connect the haptic interface and the molecule. The force (resp. position) scaling factor is denoted by $A_{f}$ (resp. $A_{d}$ ).

Depending on the manipulation mode, the user may either set the desired instantaneous position of the molecule $x_{i}$, or apply an additional force $F_{i}$ on it.

Based on the two manipulation modes described in Section 2 and the notations given above, position and force control are compared.

\subsection{Position control}

The first control scheme, the classical impedance display or Direct Force Feedback (DFF) coupling, is based on position control.

\subsubsection{The Direct Force Feedback control scheme}

The DFF coupling, represented in Figure 3, is the simplest structure to connect the haptic device to the simulation using the scaled position of the haptic handle $x_{i}$ as the input of the molecular dynamics simulator. It is also the most intuitive, since the user directly controls the position of the molecule and feels the interaction forces $F_{h}$ between the molecule and its environment. Only two scaling factors are necessary to control the molecule.

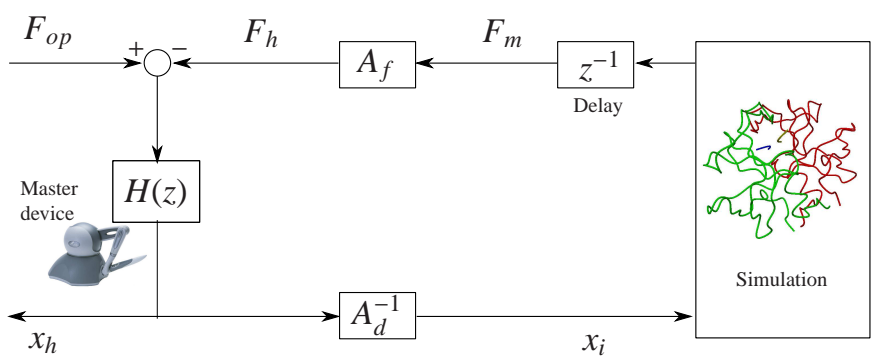

Figure 3: $D F F$ control scheme

\subsubsection{Details of the position control algorithm}

As mentioned in Section 2, the calculation of positions and forces performed by the simulation software are made on each rigid body. However, for ease of manipulation, the user manipulates the molecule as a whole, controlling the position of the center of mass $x_{i}$. As explained in Section 2.2.1, the instantaneous position $x_{i}$ of the rigid body is set by the user. Internal and external forces are computed and used to update the positions of the molecules in the environment and their internal degrees of freedom (to simulate their flexibility). This reconfiguration step involves a modification of the rigid bodies positions, and thus the actual position of the center of mass of the manipulated molecule $x_{m}$ might be different from the one set by the user using the haptic interface $x_{i}$. The user feels the force applied on the manipulated molecule $F_{m}$ composed of internal and external forces (Equation (3)). This methodology is summarized in Algorithm $1^{1}$.

The DFF coupling has one main drawback, due to the manipulation mode used. The user sets an arbitrary position using the haptic interface. The molecular system first takes the corresponding configuration before being corrected during the update step by the simulator. This can lead to instabilities, and the forces sent to the user may misrepresent the correct molecular interactions.

\subsection{Force control}

As an alternative to position control, we can consider a second manipulation mode, force control.

${ }^{1}$ For the sake of clarity only the translations are considered in this algorithm. Rotations are implemented using the same methodology. This remark also applies for Algorithm 2. 
$\overline{\text { Algorithm } 1 \text { Position control algorithm (see Section } 7 \text { for the }}$ notations)

1. Compute the desired position of the center of mass $x_{i}(k+1)$ set by the operator using the control scheme (position of the haptic handle scaled by $A_{d}$ ).

2. Set the position of the rigid bodies to $x_{i}^{j}(k+1)$, computed based on the relative positions with respect to $x_{i}$.

3. Update all interatomic forces $F_{m}^{1}(k+1), \ldots, F_{m}^{n}(k+1)$ (resp. $\left.F_{e}^{1}(k+1), \ldots, F_{e}^{q}(k+1)\right)$ applied to the rigid bodies which are inside (resp. outside) the manipulated molecule using the molecular simulator.

4. Compute the sum $F_{m}(k+1)=\sum_{j=1}^{n} F_{m}^{j}(k+1)$ of the forces applied to the molecule, and send it back to the user.

5. Update the positions $x_{m}^{j}$ (resp. $x_{e}^{l}$ ) of the rigid bodies which are inside (resp. outside) the manipulated molecule using the quasi-statics simulator. Note that in the following equations $x_{i}^{j}(k+1)\left(\right.$ resp. $F_{m}^{j}(k+1)$ and $\left.F_{e}^{l}(k+1)\right)$ are known since they have previously been computed Step 2 (resp. 3) of the algorithm:

$$
\begin{aligned}
& x_{m}^{j}(k+1)=x_{i}^{j}(k+1)+\frac{T_{m}^{2}}{m_{j}} F_{m}^{j}(k+1) \\
& x_{e}^{l}(k+1)=x_{e}^{l}(k)+\frac{T_{m}^{2}}{m_{l}} F_{e}^{l}(k+1)
\end{aligned}
$$

\subsubsection{The Force-Force control scheme}

The Force-Force $(F F)$ control scheme is depicted in Figure 4. The input of the simulation is the force applied by the user on the molecule. In addition to the scaling factors, a proportional gain $k_{i}$ is introduced. This gain is used to adjust the magnitude of the force applied to the molecule, which is based on the difference between the molecule and the haptic handle positions.

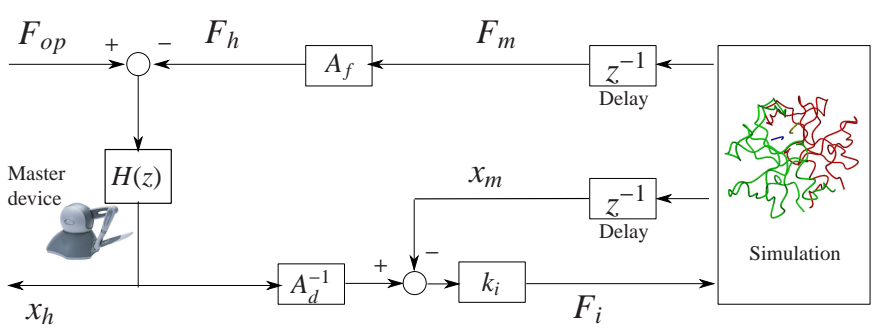

Figure 4: $F F$ control scheme

\subsubsection{Details of the force control algorithm}

Contrary to the $D F F$ algorithm, the simulator's input is the force $F_{i}$ that the user wants to apply to the entire molecule. This force is set by controlling the distance between the haptic handle and the molecule positions. The corresponding forces applied on each rigid body $F_{i}^{j}$ of the manipulated molecule as well as the environment forces are used to update the position of all the rigid bodies (inside and outside the manipulated molecule) using Equation (2). As for position control, the user feels the force applied on the manipulated molecule $F_{m}$ composed of internal and external forces (Equation (3)). This is summarized in Algorithm 2.

Algorithm 2 Force control algorithm (see Section 7 for the notations)

1. Compute the force applied by the operator using the control scheme: $F_{i}(k+1)=k_{i}\left[\frac{x_{h}(k+1)}{A_{d}}-x_{m}(k)\right]$

2. Compute the force $F_{i}^{j}(k+1)=F_{i}(k+1) / n$, applied to all the $j^{\text {th }}$ rigid bodies of the manipulated molecule.

3. Update the positions $x_{m}^{j}$ (resp. $x_{e}^{l}$ ) of the rigid bodies which are inside (resp. outside) the manipulated molecule using the quasi-statics simulator. Note that in the following equations, $F_{i}^{j}(k+1)$ is known since it has previously been computed (Step 2 of this algorithm):

$$
\begin{aligned}
& x_{m}^{j}(k+1)=x_{m}^{j}(k)+\frac{T_{m}^{2}}{m_{j}}\left(F_{m}^{j}(k)+F_{i}^{j}(k+1)\right) \\
& x_{e}^{l}(k+1)=x_{e}^{l}(k)+\frac{T_{m}^{2}}{m_{l}} F_{e}^{l}(k)
\end{aligned}
$$

4. Update all interatomic forces $F_{m}^{1}(k+1), \ldots, F_{m}^{n}(k+1)$ (resp. $\left.F_{e}^{1}(k+1), \ldots, F_{e}^{q}(k+1)\right)$ applied to the rigid bodies which are inside (resp. outside) the manipulated molecule using the molecular simulator.

5. Compute the sum $F_{m}(k+1)=\sum_{j=1}^{n} F_{m}^{j}(k+1)$ of the forces applied to the molecule, and send it to the operator.

The user input might be unrealistic in both position and force control modes. However, given the user input, its integration into the equation of motion differs. For position mode, the system configuration is first set according to the user input, and in a second step, it is corrected based on the equation of motion. On the contrary, using force control enables to integrate the user's input while solving the equation of motion (Equation (2)). The system behaves as if these forces were actually applied. Thus, it is more adapted to molecular dynamics than position control. As force mode presents a better adequacy with the solved equations, instabilities due to molecule manipulations are reduced.

\section{Comparison of transparency and stability properties}

In this section, the transparency and the sensitivity of the control schemes stability are compared. The analysis is made on one axis: scalar variables are used. The action of the operator is not taken into account, and a simplified model of the environment is considered (an equivalent spring constant $k_{e}$ ). This is obviously not enough to model the complex interactions between molecules, but comparisons of the control schemes can be made. Transparency analysis will be carried out in continuous time, without considering the delays. These assumptions are made to derive comprehensive relationships to compare the two control modes [24]. On the contrary, stability will be studied in discrete time as we will be interested on the sensitivity of the control schemes with respect to the sampling period. 


\subsection{Transparency analysis}

A haptic coupling is transparent if it perfectly renders molecular forces and molecular dynamics. It is measured by comparing the impedance of the environment $Z_{e}=F_{m} / V_{m}$ and the one felt by the operator $Z_{o p}=F_{o p} / V_{h}$ [25]. Ideal transparency is achieved when:

$$
Z_{o p}(s)=Z_{e}(s)
$$

where $s$ is the parameter of the Laplace transform. For submicron scales, the scaling factors $A_{f}$ and $A_{d}$ must be considered so that the impedances can be compared. In our context, perfect transparency will be achieved if:

$$
\frac{F_{o p}(s)}{V_{h}(s)}=\frac{A_{f}}{A_{d}} \frac{F_{m}(s)}{V_{m}(s)} \Leftrightarrow Z_{o p}(s)=\frac{A_{f}}{A_{d}} Z_{e}(s)
$$

Two distinct modes will be considered:

1. non-contact: there is no interaction between the manipulated molecule and its environment $\left(Z_{e}=0\right)$

2. contact: there are repulsive forces, modeled as a first approximation as a spring $\left(Z_{e}(s)=\frac{k_{e}}{s}\right)$

Note that, in theory, non-contact configurations do not exist in molecular systems since interactions can be felt at infinite distance (e.g. electrostatic interactions). However, molecular dynamics simulators frequently use cutoffs, i.e. distances over which interactions are considered to be zero. This makes relevant the analysis on non-contact configurations.

An approximated impedance will be derived in the frequency domain $(s=j \omega)$ considering low $(\omega<<1)$ or high $(\omega>>1)$ frequencies.

\subsubsection{The Direct Force Feedback control scheme}

Using the control scheme depicted in Figure 3, the impedance felt by the operator is computed:

$$
Z_{o p}^{D F F}(s)=\frac{F_{o p}(s)}{V_{h}(s)}=\frac{A_{f}}{A_{d}} Z_{e}(s)+\frac{1}{H(s)}
$$

Non-contact. When no force is applied to the molecule, Equation (7) is simplified to:

$$
Z_{o p}^{D F F}(j \omega)=M_{h} j \omega+B_{h}
$$

At low frequencies, the operator will mainly feel the viscosity of the haptic device. At high frequencies, the inertia of the master arm will be predominant.

Contact. When repulsive forces are applied to the manipulated molecule, the impedance felt by the operator is given by Equation (7). This corresponds to the impedance he or she should ideally feel $\left(\frac{A_{f}}{A_{d}} Z_{e}\right)$ modulated by the haptic device characteristics. For low frequencies, the impedance $Z_{o p, L F}^{D F F}$ is approximated by:

$$
Z_{o p, L F}^{D F F}(j \omega) \underset{\omega<<1}{\approx} \frac{A_{f}}{A_{d}} Z_{e}(j \omega)+B_{h}
$$

The viscosity of the haptic interface deteriorates the transparency. For high frequencies, the impedance $Z_{o p, H F}^{D F F}$ is:

$$
Z_{o p, H F}^{D F F}(j \omega) \underset{\omega>>1}{\approx} M_{h} j \omega
$$

The transparency of the coupling is only affected by the inertia of the haptic device.

\subsubsection{The Force-Force control scheme}

For the $F F$ coupling, the impedance felt by the operator is:

$$
Z_{o p}^{F F}(s)=\frac{1}{H(s)}+\frac{A_{f} k_{i}}{A_{d}\left(m s^{2}+k_{i}+k_{e}\right)} Z_{e}(s)
$$

Non-contact. As for the DFF coupling, the operator's impedance is given by Equation (8), and he or she feels the viscosity of the haptic device for low frequencies, and its inertia for high ones.

Contact. When the contact is established, $Z_{o p, L F}^{F F}$ can be computed for low frequencies:

$$
Z_{o p, L F}^{F F}(j \omega) \underset{\omega<1}{\approx} \frac{k_{i}}{k_{i}+k_{e}} \frac{A_{f}}{A_{d}} Z_{e}(j \omega)+B_{h}
$$

Equation (12) differs from the ideal impedance $\frac{A_{f}}{A_{d}} Z_{e}(j \omega)$ due to

\begin{tabular}{|c|c|c|c|c|}
\hline & \multicolumn{2}{|r|}{ Low frequencies } & \multicolumn{2}{|c|}{ High frequencies } \\
\hline & Non- & Contact & Non- & Contact \\
\hline$D F F$ & \multirow[t]{2}{*}{$B_{h}$} & $\frac{A_{f}}{A_{d}} Z_{e}(j \omega)+B_{h}$ & \multirow{2}{*}{\multicolumn{2}{|c|}{$M_{h} j \omega$}} \\
\hline$F F$ & & $\frac{k_{i}}{k_{i}+k_{e}} \frac{A_{f}}{A_{d}} Z_{e}(j \omega)+B_{h}$ & & \\
\hline
\end{tabular}
the static gain $\frac{k_{i}}{k_{i}+k_{e}}$, and the viscosity of the haptic interface. For high frequencies, the impedance felt by the user is the inertia of the haptic device.

\subsubsection{Summary}

Table 1 summarizes the approximated impedances for the two control schemes.

Table 1: Approximated values of $Z_{o p}$ for the two control schemes

For the DFF control scheme, the quality of the force rendering only depends on the haptic device characteristics. The transparency of this coupling is close to the ideal transparency. The $F F$ transparency differs from the $D F F$ one for the contact mode, for low frequencies. The ideal impedance is scaled by $\frac{k_{i}}{k_{i}+k_{e}}$ for the $F F$ coupling. To tend to perfect transparency, the value of $k_{i}$ must be greater than $k_{e}$. This means that the force applied by the user is greater than the ones coming from the molecular system. For an infinite value of $k_{i}$ the molecule position will only depend on the force applied by the user, and the manipulation mode will tend to the behavior of the position manipulation mode. On the contrary, if $k_{i}$ is much lower than $k_{e}$, the perfect impedance will be scaled by $\frac{k_{i}}{k_{e}}$. We will see in Section 5.1 that it corresponds to divide the perfect impedance by a factor less than two as long as $k_{e} \leq 100 \mathrm{~N} \cdot \mathrm{m}^{-1}$. The transparency of this coupling is thus only slightly deteriorated.

\subsection{Stability analysis}

Since passivity is conservative (it ensures stability for any operator and any environment as long as they are themselves passive) [26], it is not used in this work. We chose instead to 
compare the sensitivity of the control schemes' stability with respect to the sampling period. Thus discrete time must be used. The computational time is taken into account by introducing a one period delay on the variables computed by the simulator (see Figure 3 and Figure 4). The sensitivity analyzed is the variation of the magnitude of the roots of the characteristic equations with respect to given parameters, which is here the sampling period.

\subsubsection{Characteristic equations}

The discrete time transfer function of the DFF control scheme is derived from the coupling presented in Figure 3:

$$
\begin{aligned}
& Z\left\{\frac{x_{h}(s)}{F_{o p}(s)}\right\}=\frac{X_{h}(z)}{F_{o p}(z)} \\
& =\frac{T_{s} \frac{z-\delta}{z-1}-(1-\delta) \frac{M_{h}}{B_{h}}}{B_{h}(z-\delta)+\left[T_{s} \frac{z-\delta}{z-1}-(1-\delta) \frac{M_{h}}{B_{h}}\right] \frac{A_{f}}{A_{d}} k_{e} z^{-1}}
\end{aligned}
$$

where $Z$ is the $Z$-transform function, and $\delta$ is the parameter defined in Equation (4). The corresponding characteristic equation is:

$$
a_{3} z^{3}+a_{2} z^{2}+a_{1} z+a_{0}=0
$$

where:

$$
\begin{aligned}
& a_{3}=B_{h}, \quad a_{2}=-B_{h}(1+\delta) \\
& a_{1}=\delta B_{h}+\left(T_{s}-(1-\delta) \frac{M_{h}}{B_{h}}\right) \frac{A_{f}}{A_{d}} k_{e} \\
& a_{0}=\left(-\delta T_{s}+(1-\delta) \frac{M_{h}}{B_{h}}\right) \frac{A_{f}}{A_{d}} k_{e}
\end{aligned}
$$

The transfer function of the $F F$ coupling is computed according to Figure 4:

$$
\frac{X_{h}(z)}{F_{o p}(z)}=\frac{H(z)}{1+H(z) G(z) \frac{A_{f}}{A_{d}}}
$$

where: $G(z)=\frac{F_{m}(z)}{X_{h}(z)}=\frac{k_{i} k_{T} T_{m}^{2}}{m z(z-1)+k_{i} T_{m}^{2}+k_{e} T_{m}^{2}}$ and $m$ is the mass of the whole molecule. The characteristic equation is:

$$
b_{4} z^{4}+b_{3} z^{3}+b_{2} z^{2}+b_{1} z+b_{0}=0
$$

where:

$$
\begin{aligned}
b_{4}= & m B_{h}, \quad b_{3}=-(2+\delta) m B_{h} \\
b_{2}= & \left(k_{i}+k_{e}\right) T_{m}^{2} B_{h}+m B_{h}(1+2 \delta) \\
b_{1}= & -(1+\delta)\left(k_{i}+k_{e}\right) T_{m}^{2} B_{h}-\delta m B_{h} \\
& +\left[T_{s}-(1-\delta) \frac{M_{h}}{B_{h}}\right] k_{i} k_{e} T_{m}^{2} \frac{A_{f}}{A_{d}} \\
b_{0}= & B_{h} \delta\left(k_{i}+k_{e}\right) T_{m}^{2} \\
& +\left[-T_{s} \delta+(1-\delta) \frac{M_{h}}{B_{h}}\right] k_{i} k_{e} T_{m}^{2} \frac{A_{f}}{A_{d}}
\end{aligned}
$$

Analytical stability criteria can be obtained for both of these control schemes using the Jury criterion [22]. However, the relations derived are complex and the influence of the control scheme parameters on stability is not highlighted. In the next section, a numerical analysis is performed.

\subsubsection{Numerical comparison of the control schemes' stability}

A numerical analysis of the influence of the environment parameters $k_{e}$ and $T_{s}$ on each control scheme is performed. The variations of the highest magnitude of the roots of the characteristic equations (14) and (17) $\left(\left\|r_{\max }\right\|\right)$ are considered in Figure 5 . The numerical values correspond to the experiments carried out in Section 5.1 (see Table 3). The plots of Figure 5 represent the value of $\left\|r_{\max }\right\|$ for both the control schemes, depending on $k_{e}$ and $T_{s}$.

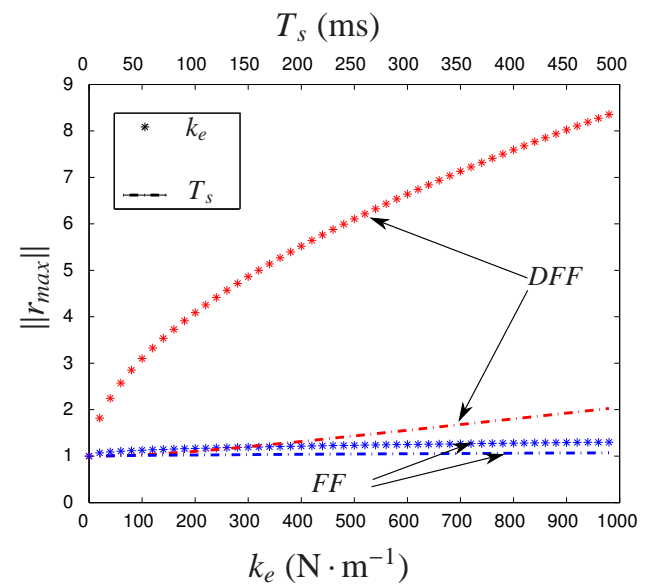

Figure 5: Highest magnitude of the roots of the characteristic equation $\left\|r_{\text {max }}\right\|$ with respect to the stiffness of the interaction $k_{e}$ and to the computation time $T_{s}$

The variations of the magnitude of the roots are of particular interest. For the two control schemes, long computations (large values of $T_{s}$ ) and/or stiff environments may lead to an unstable system. Moreover the magnitude of the roots of the $D F F$ control scheme quickly increases when $T_{s}$ or $k_{e}$ are increased. On the contrary, the roots obtained with the $F F$ control scheme are almost constant. Thus, the $D F F$ control scheme stability is more sensitive to the variations of $T_{s}$ than the $F F$ one. These results will be experimentally confirmed in the next section.

\subsection{Comparison of the control schemes' specificity}

As seen from Section 2 to Section 4, the position and force control modes present different characteristics in terms of adequacy with the algorithm, transparency and stability, which are summarized in Table 2. Position control is more transparent. However, force control is better suited for the integration into the simulator since the user's input is directly taken into account while solving the equation of motion, and its stability is less sensitive to environmental conditions.

Next sections detail the possible applications of our system, and highlight the advantages and drawbacks of both position and control mode depending on the given task.

\section{Molecular manipulations and haptic feedback of inter- action phenomena}

As seen in Section 1, a wide variety of applications must be considered. To illustrate the possibilities of our system, the following experiments are performed in the next sections: 
Table 2: Comparison of the $D F F$ and $F F$ coupling schemes

\begin{tabular}{|c|c|c|}
\hline & Advantages & Drawbacks \\
\hline$D F F$ & Good transparency & $\begin{array}{l}\text { - Molecule's control not } \\
\text { optimal for the simulation } \\
\text { - Stability sensitive to en- } \\
\text { vironmental conditions }\end{array}$ \\
\hline$F F$ & $\begin{array}{l}\text { - Molecule's control } \\
\text { suited for the simulation } \\
\text { - Good transparency } \\
\text { during non-contact } \\
\text { - Stability not sensitive to } \\
\text { environmental conditions }\end{array}$ & $\begin{array}{l}\text { Ideal transparency scaled } \\
\text { by } \frac{k_{i}}{k_{e}+k_{i}} \text { during contact }\end{array}$ \\
\hline
\end{tabular}

- manipulation of whole molecules to modify the global structure of the molecular system,

- deformation of molecular structures to measure their inherent properties,

- determination of equilibrium positions which is a common example of the specific phenomena of nanoscales that users would like to understand.

Experiments with varying molecules and parameters have been carried out. For the sake of clarity, only some of them which illustrate the three types of operation listed above are presented. Similar conclusions were derived for the other cases we considered.

The experiments are performed on a $3 \mathrm{GHz}$ Intel PC processor, using a Phantom Omni haptic interface from Sensable ${ }^{2}$. This master arm is a 6 degrees of freedom (dof) movement and a 3 dof force-feedback device, with an inertia $M_{h}=0.072 \mathrm{~kg}$ and a viscosity $B_{h}=0.005 \mathrm{~N} . \mathrm{s} . \mathrm{m}^{-1}$ [23]. The maximum force that it can provide is $3.3 \mathrm{~N}$. In the following experiments, the maximum force sent to the haptic interface is limited to $3 \mathrm{~N}$. The OpenHaptics development toolkit provided by Sensable is used to communicate with the haptic interface. The setup is presented in Figure 6.

\subsection{Manipulation of whole molecules, the HIV example}

The haptic device must enable the user to manipulate the whole molecule into the environment, so that it would be equivalent for him or her to manipulate their center of mass in the case of rigid molecules. This mode has been chosen since it enables an untrained user to precisely and intuitively move the molecules. Two solutions are possible. The first one is to set the position of its center of mass, and the second one is to apply a force on each of the rigid bodies. To compare these two manipulation modes and the associated control schemes, the HIV protease and its inhibitors (Protein Data Bank code: 2AZ8) is considered. This system is composed of 848 degrees of freedom. The parameters used for the simulator and the haptic coupling schemes are summarized in Table 3 . These values are the ones used for the numerical comparison of stability in Section

${ }^{2} \mathrm{http}: / /$ www.sensable.com

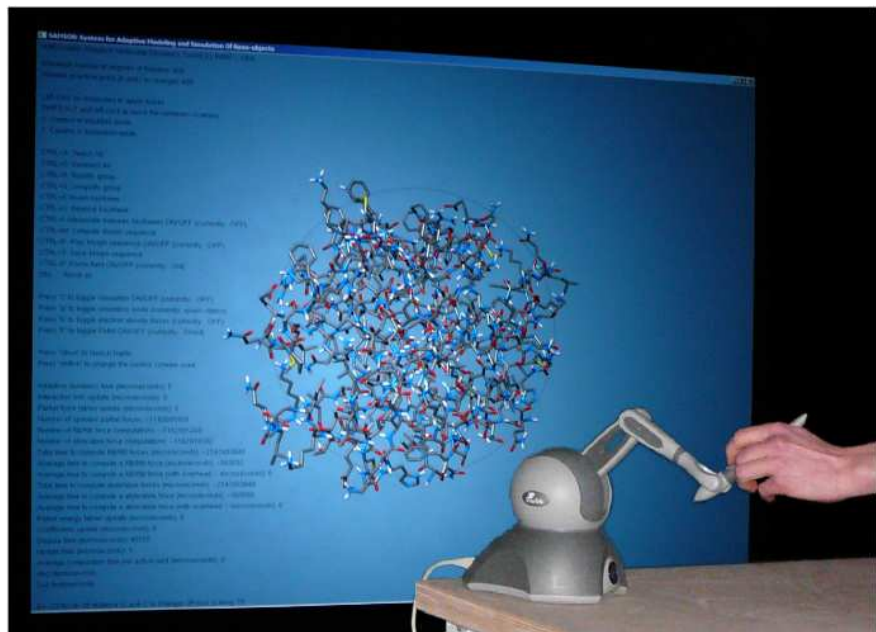

Figure 6: The HIV molecule and its inhibitors, 848 degrees of freedom. A haptic device is used to control the inhibitors and to feel the interactions with the HIV molecule.

4.2. The experiment consists in extracting an inhibitor of the molecule, and then reinserting it. All the molecules are flexible.

Table 3: Parameters of the simulator and haptic coupling schemes for the HIV experiment

\begin{tabular}{|l|l|}
\hline \multirow{4}{*}{ Simulator } & - Mass of the manipulated molecule: \\
& $m=7.5 \cdot 10^{-25} \mathrm{~kg}$ \\
& - Cut off distance: $d=8 \AA$ \\
& - Integration time for the equation of motion: \\
& $T_{m}=2 \cdot 10^{-15} \mathrm{~s}$. \\
\hline \multirow{2}{*}{ Haptic } & - Force scaling coefficient: $A_{f}=0.3 \cdot 10^{9}$ \\
coupling & - Displacement scaling coefficient: $A_{d}=0.25 \cdot 10^{9}$ \\
& - Stiffness of the $F F$ coupling: $k_{i}=100 \mathrm{~N} \cdot \mathrm{m}^{-1}$ \\
\hline
\end{tabular}

The experiment is repeated twice for both the $D F F$ and $F F$ coupling schemes, with varying number of activated dof (172 for the first experiment, 848 for the second one), and thus varying simulation periods ( $T_{s}=92$ and $117 \mathrm{~ms}$ ). This is possible thanks to the adaptive simulation algorithms used by our simulator which enable to decrease the number of active degrees of freedom with a minimal loss of accuracy [19].

In order to compare the results, the movement of the user has been limited to one axis (with 3D force feedback). The forces returned to the operator on the $y$ axis of the haptic interface with respect to the position of the molecule's center of mass are depicted in Figure 7 and 8.

Three phases can clearly be distinguished. The first one is the extraction of one of the inhibitors, when it is situated at less than $16 \AA$ of its initial position. The second phase consists in manipulating the inhibitor away from the rest of the structure, when the inhibitor is further than $16 \AA$. No force is applied on it. The reinsertion into the system occurs when the position is again less than $16 \AA$.

The forces felt using the $D F F$ and $F F$ control schemes are sim- 


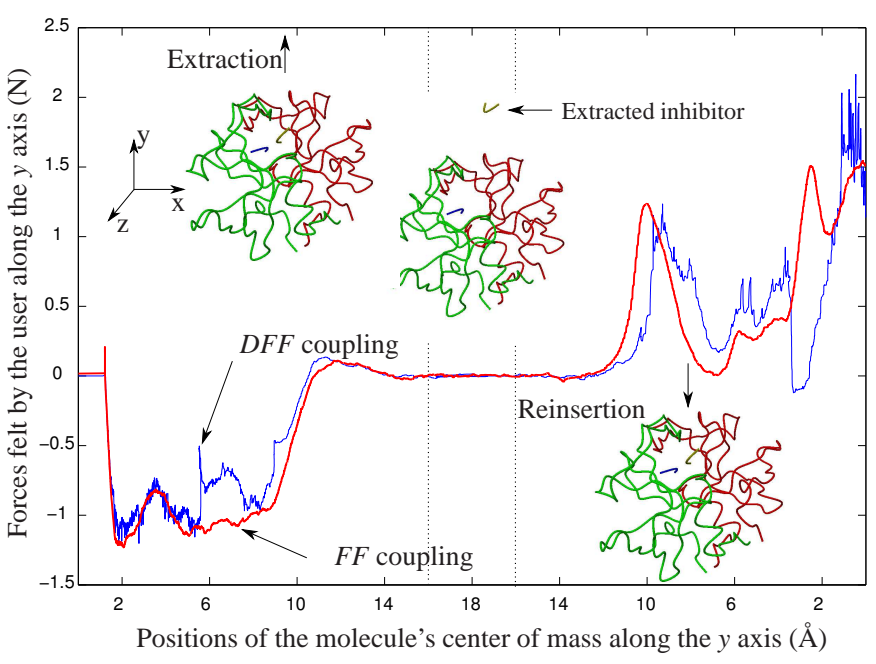

Figure 7: Force felt by the user while manipulating an HIV's inhibitor (interaction forces between molecules). 172 dof activated $-T_{s}=92 \mathrm{~ms}$

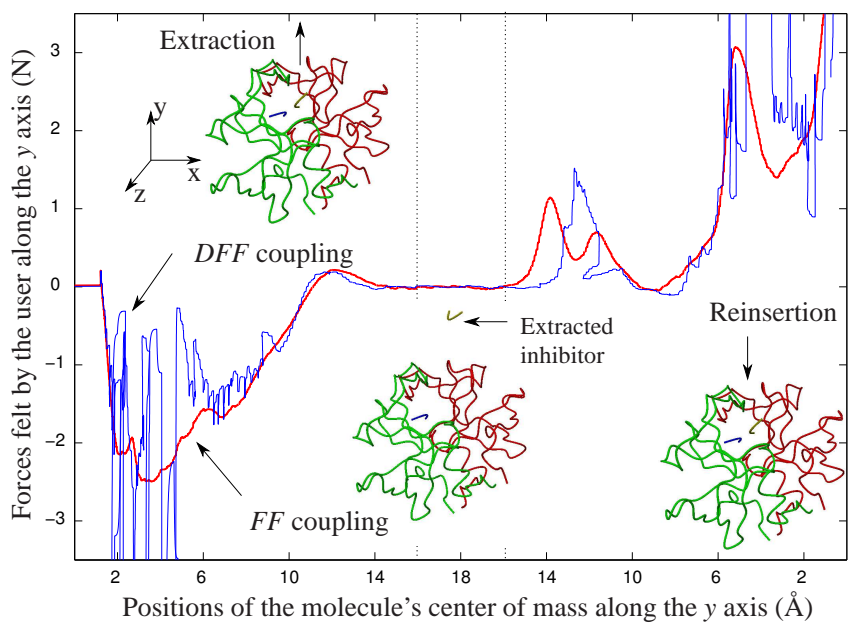

Figure 8: Force felt by the user while manipulating an HIV's inhibitor (interaction forces between molecules). All the 848 dof activated $-T_{S}=117 \mathrm{~ms}$

ilar. For the two coupling schemes, the magnitude of the haptic forces are in the order of the newton so that the interaction forces can clearly be felt by the user. The differences on the force profile are due to the flexibility of the molecules. Depending on the manipulation conditions (velocity of the molecule, force exerted, etc.) the deformations are not the same, leading to slightly different configurations and interaction forces.

As predicted in Section 4.2, the DFF coupling is very sensitive to the environment characteristics. Only a few oscillations can be seen in Figure 7 for very stiff interactions. When the sampling period increases, oscillations become important and more frequent. On the opposite, the $F F$ coupling remains stable even for stiff environments, and with long simulation periods. Adding damping to the $D F F$ coupling would improves the stability, but transparency would be deteriorated. Since molecular interactions involve complex variation of the forces and efforts of small magnitude (in particular for attractive interactions), transparency is a major concern to provide to the user a high fidelity force feedback.

\subsection{Deformation of molecular structures}

The measurement of the molecule inherent properties (stiffness, inertia, ...) is of primary importance [1]. To deform such structures, it is necessary to apply an external force on the molecule. This is done using force control.

The experiment presented in this paragraph consists in manipulating a single rigid body of a flexible molecule using the $F F$ coupling, and feeling the internal forces and the inertia. The same methodology as the one described in Algorithm 2 is used, except that the whole molecule is replaced by a single rigid body $\mathcal{R}_{m}^{j}$ and the kinematic constraint forces are added as mentioned in Section 2. Since no other molecules are present, the external force applied to the rigid body are null $\left(F_{\text {ext }}=0\right)$, and the operator will only feel the internal forces $F_{m}=F_{\text {int }}$. Using the haptic device the operator applies a force on one of the rigid bodies of the HIV inhibitor to unfold it. The other extremity is fixed. When the molecule is stretched, the user moves back the haptic device to the initial position. Doing so, the molecule is refolded.

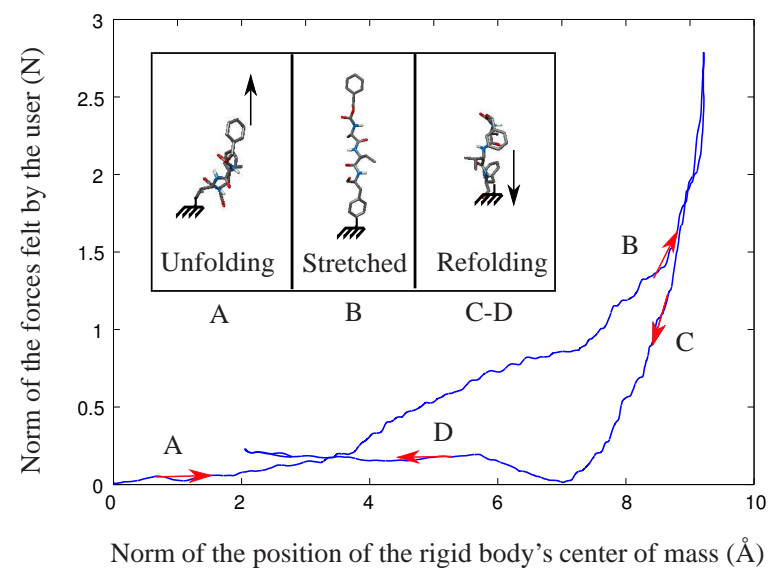

Figure 9: Forces felt by the user while unfolding an inhibitor of the HIV virus (A), keeping the molecule stretched (B), and refolding it (C and D). The user feels the internal forces. 17 degrees of freedom, $T_{s}=14 m s, A_{d}=5 \cdot 10^{9}$, $A_{f}=0.1 \cdot 10^{9}, k_{i}=20 \mathrm{~N} . \mathrm{m}^{-1}$.

Figure 9 represents the norm of the forces felt by the user against the norm of the position of the center of mass. The forces increase since the molecule is stretched (part A). When the molecule is deployed, the user applies an additional force and the internal forces increase (part B). After that, the user moves back the rigid body and he or she releases the constraint applied on it. The forces quickly decrease because of the reduction of internal forces (part C). Finally, the molecule is moved toward its initial position. The interaction forces due to the other rigid bodies are felt (part D). This shape is consistent with the one described in [1]. Our system is able to render small variations of the forces to the user (in the range of the nanonewton). The FF coupling is well suited to deform molecules by applying an external force, and can be used to measure their inherent properties. 


\subsection{Determination of equilibrium positions}

Using force control, the user applies an effort on the manipulated molecule, and the equilibrium state of the system takes into account this external force. However, to better understand molecular interactions, it is necessary to know the equilibrium state around a given position without considering the action of the user. Position control with force feedback enables the user to get such a knowledge.

An experiment involving two water molecules is performed using the DFF coupling. Due to van der Waals forces, there is an equilibrium position when the distance of the two water molecules is around 2.6. Attractive and repulsive forces tend to keep the molecules in this position.

In the following experiment, one of the molecules is fixed. The user manipulates the second one around the equilibrium position, while feeling the interaction forces which tend to keep the system into the equilibrium state. Experimental results are given in Figure 10. Position and force are plotted with respect to the time to evaluate the time taken by the system to reach the equilibrium position.

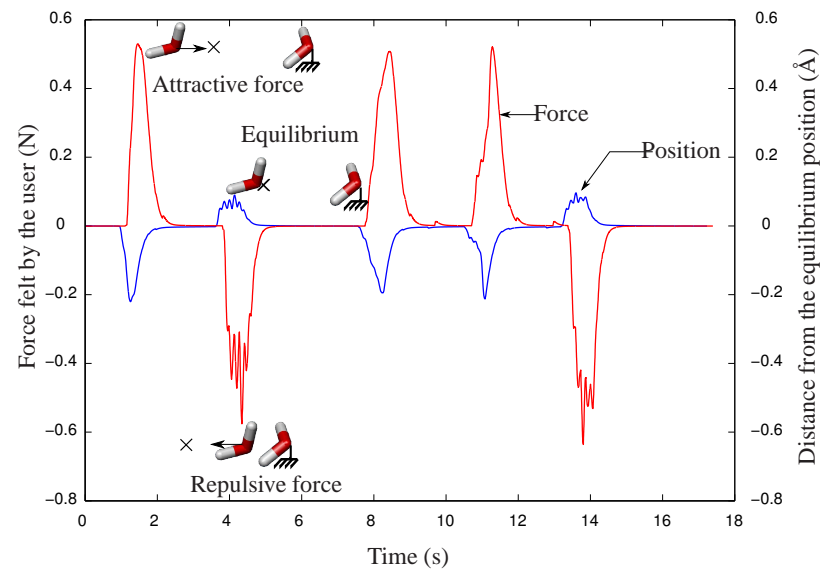

Figure 10: Manipulation of two water molecules. The black cross indicates the position of the equilibrium position. $T_{s}=18 \mathrm{~ms}, A_{d}=0.25 \cdot 10^{9}, A_{f}=$ $1.5 \cdot 10^{9}$.

The user can feel both attractive and repulsive forces and can clearly determine the equilibrium position since it corresponds to a null force feedback. This helps him or her understand one of the most specific molecular interactions, the long range attractive and repulsive phenomena.

\section{Conclusion}

In this work, we have presented a new tool for the analysis of molecular structures. The simulator computes the reconfiguration of molecular systems composed of hundreds or thousands of degrees of freedom in a few tenths of a second. Thanks to its opened architecture, the haptic coupling can directly be integrated into the equation of motion, leading to two possible control modes. The associated $D F F$ and $F F$ haptic coupling schemes are complementary since high transparency is achieved for position control. Force control presents a better adequacy to molecular dynamics and its stability is not sensitive to the environmental conditions.

Based on the control mode specificity, different operations can be carried out. Position control enables to get a better understanding of nanoscale interactions. Force control is used to deform molecular structures to measure their inherent properties. The performed experiments are very promising since they highlight the diversity of applications that can be handled by our system. The detailed analysis of the properties of the control modes can also easily be used to implement haptic feeback on other molecular simulators.

The stability conditions are not easily verified. Based on the experimental results, it can be seen however that force control remains stable for given values of the control scheme parameters. This is possible since its stability is not sensitive to environmental conditions. Future work include determining precisely how to choose the parameters to get a stable system. As position control stability is sensitive, non-constant gain might be a solution to get a stable system for a wide variety of environmental conditions. A user study will then be conducted to determine the benefits of the different haptic feedbacks from the two control modes.

To enable the design of new molecular structures, our tool should offer the possibility to add and/or remove atoms. Haptic feedback would in this case assist the user while breaking the energy barriers. This functionality will be investigated.

\section{Notations}

\begin{tabular}{rl|l}
$F_{o p} / F_{h}$ & User/haptic force \\
$x_{h} / v_{h}$ & Haptic position/velocity \\
Haptic. $\quad H(s) / H(z)$ & Haptic device continuous/discrete transfer \\
$M_{h} / B_{h}$ & function \\
Haptic device inertia/viscosity
\end{tabular}

\begin{tabular}{r|ll} 
& $A_{f} / A_{d}$ & Force/position scaling factor \\
Coupling. $\quad k_{i}$ & Stiffness of the $F F$ coupling \\
$T_{s}$ & Sampling period \\
$T_{m}$ & Simulation integration period \\
$x_{m} / x_{m}^{j}$ & Molecule $/ j^{\text {th }}$ rigid body actual position \\
$x_{i} / x_{i}^{j}$ & Molecule $/ j^{\text {th }}$ rigid body input position \\
$F_{m} / F_{m}^{j}$ & Force applied to the molecule $/ j^{t h}$ rigid body \\
$F_{i} / F_{i}^{j}$ & User force applied to the molecule $/ j^{\text {th }}$ rigid \\
bolecule. \\
$x_{e}^{l} / F_{e}^{l}$ & $\begin{array}{l}\text { Position } / \text { force of rigid bodies in the envi- } \\
\text { ronment } \\
m / m_{p}\end{array}$ & Molecule $/ p^{t h}$ rigid body inertia
\end{tabular}

\section{Acknowledgment}

This work was supported by the French National Agency of Research, through the PACMAN project. 


\section{References}

[1] M. Hamdi, A. Ferreira, G. Sharma, C. Mavroidis, Prototyping bionanorobots using molecular dynamics simulation and virtual reality, Microelectronics Journal 39 (2) (2008) 190-201.

[2] M. Moore, A. Enomoto, T. Nakano, Y. Okaie, T. Suda, Interfacing with nanomachines through molecular communication, in: IEEE International Conference on Systems, Man and Cybernetics, 2007, pp. 18-23.

[3] B. Atakan, O. Akan, An information theoretical approach for molecular communication, Bio-Inspired Models of Network, Information and Computing Systems (2007) 33-40.

[4] D. D. Rossi, A. Ahluwalia, M. Mule, Molecular and supramolecular systems for sensing and actuation, IEEE Engineering in Medicine and Biology Magazine 13 (1) (1994) 103-111.

[5] T. Grove, K. Puckett, N. Brunet, G. Mihajlovic, L. McFadden, X. Peng, S. von Molnar, T. Moerland, P. Chase, Packaging actomyosin-based biomolecular motor-driven devices for nanoactuator applications, IEEE Transactions on Advanced Packaging 28 (4) (2005) 556-563.

[6] P. Persson, M. Cooper, L. Tibell, S. Ainsworth, A. Ynnerman, B.-H. Jonsson, Designing and evaluating a haptic system for biomolecular education, in: IEEE Virtual Reality Conference, 2007, pp. 171-178.

[7] J. Murayama, H. Shimizu, C. S. Nam, H. Satoh, M. Sato, An educational environment for chemical contents with haptic interaction, in: Proceedings of the International Conference on Cyberworlds, 2007, pp. 346-352.

[8] G. Sankaranarayanan, S. Weghorst, M. Sanner, A. Gillet, A. Olson, Role of haptics in teaching structural molecular biology, in: Proceedings of the 11th Symposium on Haptic Interfaces for Virtual Environment and Teleoperator Systems, 2003, pp. 363-366.

[9] F. P. Brooks, Jr., M. Ouh-Young, J. J. Batter, P. Jerome Kilpatrick, Project GROPE - Haptic displays for scientific visualization, in: Proceedings of the 17th annual conference on Computer graphics and interactive techniques, 1990, pp. 177-185.

[10] M. Hamdi, G. Sharma, A. Ferreira, C. Mavroidis, Molecular mechanics simulation of bio-nanorobotic components using force feedback, in: IEEE International Conference on Robotics and Biomimetics, 2005, pp. 105110 .

[11] L. Dominjon, A. Lécuyer, J.-M. Burkhardt, G. Andrade-Barroso, S. Richir, The "Bubble" technique: interacting with large virtual environments using haptic devices with limited workspace, in: Proceedings of the First Joint Eurohaptics Conference and Symposium on Haptic Interfaces for Virtual Environment and Teleoperator Systems, 2005, pp. 639-640.

[12] E. Subasi, C. Basdogan, A new haptic interaction and visualization approach for rigid molecular docking in virtual environments, Presence: Teleoperators and Virtual Environments 17 (1) (2008) 73-90.

[13] Y.-G. Lee, K. W. Lyons, Smoothing haptic interaction using molecular force calculations, Computer-Aided Design 36 (1) (2004) 75 - 90.

[14] B. Daunay, S. Régnier, Stable six degrees of freedom haptic feedback for flexible ligand-protein docking, Computer Aided Design 41 (12) (2009) 886-895.

[15] A. M. Wollacott, K. M. Merz Jr., Haptic applications for molecular structure manipulation, Journal of Molecular Graphics and Modelling 25 (6) (2007) $801-805$.

[16] J. E. Stone, J. Gullingsrud, K. Schulten, A system for interactive molecular dynamics simulation, in: Proceedings of the symposium on Interactive 3D graphics, 2001, pp. 191-194.

[17] O. Delalande, N. Frey, G. Grasseau, M. Baaden, Complex molecular assemblies at hand via interactive simulations, Journal of Computational Chemistry 30 (15) (2009) 2375-2387.

[18] A. Bolopion, B. Cagneau, S. Redon, S. Régnier, Haptic feedback for molecular simulation, in: Proceedings of the IEEE International Conference on Intelligent Robots and Systems, 2009, pp. 237-242.

[19] R. Rossi, M. Isorce, S. Morin, J. Flocard, K. Arumugam, S. Crouzy, M. Vivaudou, S. Redon, Adaptive torsion-angle quasi-statics: a general simulation method with applications to protein structure analysis and design, Bioinformatics 23 (13) (2007) i408-417.

[20] S. Grudinin, S. Redon, Practical modeling of molecular systems with symmetries, Journal of Computational Chemistry (2010) to appear.

[21] R. Featherstone, A divide-and-conquer articulated-body algorithm for parallel $\mathrm{O}(\log (\mathrm{n}))$ calculation of rigid-body dynamics. Part 1: basic algorithm, The International Journal of Robotics Research 18 (9) (1999) $867-875$.

[22] K. Ogata, Discrete-time control systems, $2^{\text {nd }}$ Edition, Prentice Hall, 1995.
[23] N. Diolaiti, G. Niemeyer, F. Barbagli, J. Salisbury, Stability of haptic rendering: Discretization, quantization, time delay, and coulomb effects, IEEE Transactions on Robotics 22 (2) (2006) 256-268.

[24] A. Bolopion, B. Cagneau, D. S. Haliyo, S. Régnier, Analysis of stability and transparency for nanoscale force feedback in bilateral coupling, Journal of Micro-Nano Mechatronics 4 (4) (2008) 145-158.

[25] D. Lawrence, Stability and transparency in bilateral teleoperation, IEEE Transactions on Robotics and Automation 9 (5) (1993) 624-637.

[26] H. K. Khalil, Nonlinear systems, $3^{\text {rd }}$ Edition, Prentice Hall, 2002. 